\title{
ERGODIC ACTIONS OF UNIVERSAL QUANTUM GROUPS ON OPERATOR ALGEBRAS
}

\author{
SHUZHOU WANG
}

\begin{abstract}
We construct ergodic actions of compact quantum groups on $C^{*}$-algebras and von Neumann algebras, and exhibit phenomena of such actions that are of different nature from ergodic actions of compact groups.

In particular, we construct: (1). an ergodic action of the compact quantum $A_{u}(Q)$ on the type $\mathrm{III}_{\lambda}$ Powers factor $R_{\lambda}$ for an appropriate positive $Q \in G L(2, \mathbb{R}) ;(2)$. an ergodic action of the compact quantum group $A_{u}(n)$ on the hyperfinite $\mathrm{II}_{1}$ factor $R$; (3). an ergodic action of the compact quantum group $A_{u}(Q)$ on the Cuntz algebra $\mathcal{O}_{n}$ for each positive matrix $Q \in G L(n, \mathbb{C}) ;(4)$. ergodic actions of compact quantum groups on the their homogeneous spaces, as well as an example of a non-homogeneous classical space that admits an ergodic action of a compact quantum group.
\end{abstract}

\section{INTRODUCTION}

It is well known that compact groups admit no ergodic actions on operator algebras other than the finite ones (i.e. those with finite traces) [15]. Therefore, there arosed the following basic problem (cf p76 of [15]): Construct an ergodic action of a semisimple compact Lie group on the Murray-von Neumann $\mathrm{II}_{1}$ factor $R$. Later, Wassermann developed some general theory of ergodic actions of compact groups on operator algebras and showed that $S U(2)$ cannot act ergodically on $R$ [33, 34], leaving experts the doubt that semisimple compact Lie groups admit ergodic actions on $R$ at all. In [5], Boca studied the general theory of ergodic action of compact quantum groups [37] on $C^{*}$-algebras and generalized some basic results on ergodic actions of compact groups to compact quantum groups. But so far there is still a lack of non-trivial examples of ergodic actions of compact quantum groups on operator algebras.

1991 Mathematics Subject Classification. Primary 46L55, 46L89; Secondary 16W30, 81R50.

Key words and phrases. Quantum groups, ergodic actions, $C^{*}$-algebras, von Neumann algebras. 
The purpose of the present paper is two-fold, which is in some sense opposite to that of Boca [5]. First, we show that some new phenomena can occur for ergodic actions of quantum groups. Second, we supply some general methods to construct ergodic actions of compact quantum groups on operator algebras and give several non-trivial examples of such actions. We show that the universal compact matrix quantum groups $A_{u}(Q)$ of [27, 28 admit ergodic actions on both the (infinite) injective factors of type III (for $Q \neq c I_{n}, c \in \mathbb{C}^{*}$ ) and the (infinite) Cuntz algebras (for $Q>0$ ). We construct an ergodic action of the universal compact matrix quantum group of Kac type $A_{u}(n)$ on the hyperfinite factor $R$, which may not admit ergodic actions of any semisimple compact Lie group [34]. We also study ergodic actions of compact quantum groups on their homogeneous spaces and show that there are non-homogeneous classical spaces that admit ergodic actions of quantum groups. These results show that compact quantum groups have a much richer theory of ergodic actions on operator algebras than compact (Lie) groups.

Unlike Boca [5], we study actions of compact quantum groups on both $C^{*}$-algebras and von Neumann algebras, not just $C^{*}$-algebras. Our construction of ergodic actions of compact quantum groups on von Neumann algebras come from their "measure preserving" actions on $C^{*}$-algebras, just as in the classical situation (see Theorem 2.5). One of our constructions of ergodic actions (see Sect. 3) uses tensor products of irreducible representations of compact quantum groups. This method was first used by Wassermann [35] in the setting of Lie groups (instead of quantum groups) to construct subfactors from their "product type actions". At the other extreme, actions of quantum groups with large fixed point algebras (i.e. prime actions) have been studied by many authors, see, e.g. [9, 7]. Generalizing the canonical action of compact Lie groups on the Cuntz algebras [8] introduced by Doplicher-Roberts [12, 10], Konishi et al [19] study (the non-ergodic) action of $S U_{q}(2)$ on the Cuntz algebra $\mathcal{O}_{2}$ and its CAR subalgebra and show that their fixed point algebras coincide (see also [20]). This result is extended to $S U_{q}(n)$ by Paolucci [22]. In [21], this action of the quantum group $S U_{q}(n)$ is induced to a (non-ergodic) action on the Powers factor $R_{\lambda}$ by a rather complicated method, which follows from our result Theorem 2.5 in a much simpler and more conceptual manner.

The contents of this paper are as follows. In 32 , we give a general method of construction of quantum group actions on von Neumann algebras from their "measure preserving" action on $C^{*}$-algebras. Using this and a result of Banica [3] on the tensor products of the fundamental 
representation of $A_{u}(Q)$, we construct in $\$ 3$ an ergodic action of a universal quantum groups $A_{u}(Q)$ on the Powers factor $R_{\lambda}$ of type $\mathrm{III}_{\lambda}$ and and an ergodic action of $A_{u}(n)$ on the hyperfinite $\mathrm{II}_{1}$ factor $R$. In \$1, using results of Banica [2], we show that the fixed point subalgebra of $R$ under the quantum subgroup $A_{o}(n)$ of $A_{u}(n)$ is also a factor and that the action of $A_{o}(n)$ on $R$ is prime. In $\$ 5$, we construct ergodic action of $A_{u}(Q)$ on the Cuntz algebras and on the injective factor $R_{\infty}$ of type $\mathrm{III}_{1}$ as well as the other factors of type III. It is also shown that the (unimodular) compact quantum group $A_{u}(n)$ of Kac type acts ergodically on the injective factor of type $\mathrm{III}_{\frac{1}{n}}$, a fact rather surprising to us. In the last section $\$$, we study ergodic actions of compact quantum groups on their "quotient spaces", and show that the quantum automorphism group $A_{\text {aut }}\left(X_{4}\right)$ acts ergodically on the classical space $X_{4}$ with 4 points, but $X_{4}$ is not isomorphic to a quotient space. We point out that instead of using the fundamental representation of $A_{u}(Q)$, we can also use representations of free products of compact quantum groups [28] in the examples in \$3 and \$5 for the constructions of ergodic actions.

\section{Lifting aCtions on $C^{*}$-Algebras to von Neumann}

\section{ALGEBRAS}

In this section, we describe (Theorem 2.5) how to construct ergodic actions of compact quantum groups on von Neumann algebras from "measure preserving" actions on noncommutative topological spaces (i.e. $C^{*}$-algebras).

To fix notation, we first recall some basic notions concerning actions of quantum groups on operator algebras ([1, 5, 23, 32]). For convenience in this paper, we will use the definition given in [32] for the notion of actions of compact quantum groups on $C^{*}$-algebras. As in [32, Woronowicz Hopf $C^{*}$-algebras are assumed to be full in order to define morphisms. We adapt the following convention (see [28, 27, 32]): when $A=C(G)$ is a Woronowicz Hopf $C^{*}$-algebra, we also say that $A$ is a compact quantum group, referring to the dual object $G$.

Definition 2.1. ( $c f$ [32]) A (left) action of a compact quantum group $A$ on a $C^{*}$-algebra $B$ is a unital *-homomorphism $\alpha$ from $B$ to $B \otimes A$ such that

(1). $\left(i d_{B} \otimes \Phi\right) \alpha=\left(\alpha \otimes i d_{A}\right) \alpha$, where $\Phi$ is the coproduct on $A$;

(2). $\left(i d_{B} \otimes \epsilon\right) \alpha=i d_{B}$, where $\epsilon$ is the counit on $A$;

(3). There is a dense ${ }^{*}$-subalgebra $\mathcal{B}$ of $B$, such that

$$
\alpha(\mathcal{B}) \subseteq \mathcal{B} \otimes \mathcal{A},
$$


where $\mathcal{A}$ is the canonical dense ${ }^{*}$-subalgebra of $A$.

Remarks. (1). The definition above is equivalent to the one in Podles [23]. As in [23], we do not impose the condition that $\alpha$ is injective, which is required in [1, [], though the examples constructed in this paper satisfy this condition. We conjecture that this condition is a consequence of the other conditions in the definition. A special case of this conjecture says that the coproduct of a Woronowicz Hopf $C^{*}$ algebra is injective, which is true for both the full Woronowicz Hopf $C^{*}$-algebras (because of the counital property) and the reduced ones (because of Baaj-Skandalis [1]). Even if this conjecture is false, one can still obtain an injective $\tilde{\alpha}$ from $\alpha$ by passing to the quotient of $B$ by the kernel of $\alpha$. We leave the verification of the latter as an exercise for the reader.

(2). The above notion of left action of quantum group $G$ would be called right coaction of the Woronowicz Hopf $C^{*}$-algebra $C(G)$ by some other authors. But we prefer the more geometric term "action of quantum group". We can similarly define a right action of a quantum group $G$, which would be called a left coaction of the Woronowicz Hopf $C^{*}$-algebra $C(G)$ by some other specialists.

Definition 2.2. Let $\alpha$ be an action of a compact quantum group $A$ on $B$. An element $b$ of $B$ is said to be fixed under $\alpha$ (or invariant under $\alpha$ ) if

$$
\alpha(b)=b \otimes 1_{A} .
$$

The fixed point algebra $B^{\alpha}$ (or $B^{A}$ if no confusion arises) of the action $\alpha$ is

$$
B^{\alpha}=\left\{b \in B \mid \alpha(b)=b \otimes 1_{A}\right\} .
$$

The action of $A$ is said to be ergodic if $B^{\alpha}=\mathbb{C} I$. A continuous functional $\phi$ on $B$ is said to be invariant under $\alpha$ if

$$
\left(\phi \otimes i d_{A}\right) \alpha(b)=\phi(b) 1_{A} .
$$

Fix an action $\alpha$ of a compact quantum group $A$ on $B$. Let $h$ be the Haar state on $A$ [37, 28, 26]. Then we have

Proposition 2.3. (1). The map $E=(1 \otimes h) \alpha$ is a projection of norm one from $B$ onto $B^{\alpha}$;

(2). Let

$$
\mathcal{B}^{\alpha}=\left\{b \in \mathcal{B} \mid \alpha(b)=b \otimes 1_{A}\right\} .
$$

Then $\mathcal{B}^{\alpha}$ is norm dense in $B^{\alpha}$. Hence the action $\alpha$ is ergodic if and only if it is so when restricted to the dense ${ }^{*}$-subalgebra $\mathcal{B}$ of $B$. 
Proof. (1). This is an easy consequence of the following form of the invariance of the Haar state (cf [37]):

$$
\left(i d_{A} \otimes h\right) \Phi(a)=h(a) 1_{A}, \quad a \in A .
$$

(2). If $b \in B^{\alpha}$, then $b$ can be approximated in norm by a sequence of elements $b_{l} \in \mathcal{B}$. Let $\bar{b}_{l}$ be the average of $b_{l}$ :

$$
\bar{b}_{l}=\left(1_{B} \otimes h\right) \alpha\left(b_{l}\right) .
$$

Then from part (1) of the proposition, $\bar{b}_{l} \in B^{\alpha}$. From condition (3) of Definition 2.1, we see that $\bar{b}_{l} \in \mathcal{B}^{\alpha}$. Moreover,

$$
\begin{aligned}
\left\|\bar{b}_{l}-b\right\| & =\left\|(1 \otimes h) \alpha\left(b_{l}-b\right)\right\| \\
& \leq\|(1 \otimes h) \alpha\|\left\|b_{l}-b\right\| \rightarrow 0 .
\end{aligned}
$$

The rest is clear.

Q.E.D.

Preserve the notation above. Let $\mathfrak{A}$ be the von Neumann algebra generated by the GNS representation $\pi_{h}$ of $A$ for the state $h$. Then $\mathfrak{A}$ is a Hopf von Neumann algebra. For later use, we need to adapt the definitions above to the situation of von Neumann algebras.

Definition 2.4. A right coaction of a Hopf von Neumann algebra $\mathfrak{A}$ on a von Neumann algebra $\mathfrak{B}$ is a normal homomorphism $\alpha$ from $\mathfrak{B}$ to $\mathfrak{B} \otimes \mathfrak{A}$ such that

(1). $\left(i d_{\mathfrak{B}} \otimes \Phi\right) \alpha=\left(\alpha \otimes i d_{\mathfrak{A}}\right) \alpha$, where $\Phi$ is the coproduct on $\mathfrak{A}$;

(2). $\alpha(\mathfrak{B})(1 \otimes \mathfrak{A})$ generates the von Neumann algebra $\mathfrak{B} \otimes \mathfrak{A}$.

The main reason why we use the term "coaction of Hopf von Neumann algebra" is that von Neumann algebras are measure-theoretic objects instead of geometric-topological objects (cf. Remark (2) after Definition 2.1). Condition (2) in the above definition is an analog of the density condition as used in the Hopf $C^{*}$-algebra setting [1, 23]. It is well known that there is no analogue of counit in the Hopf von Neumann algebra situation simply because a von Neumann algebra corresponds to a measure space in the commutative case (the simplest case), and functions are defined only up to sets of measure zero. Hence we do not have an analog of condition (2) of Definition 2.1 for Hopf von Neumann algebras coactions.

If $\mathfrak{A}$ comes from the GNS-representation of the Haar state on a compact quantum group $A$ and $\mathfrak{A}$ coacts on the right on some von Neumann algebra $\mathfrak{B}$, we will abuse the terminology by saying that the quantum group $A$ acts on $\mathfrak{B}$. Other notions such as invariant elements (or functionals), fixed point algebra and ergodic actions in the $C^{*}$-case above can also be carried over to to the von Neumann algebra situation. 
The main result of this section is the following

Theorem 2.5. Let $B$ be a $C^{*}$-algebra endowed with an action $\alpha$ of a compact quantum group $A$. Let $\tau$ be an $\alpha$-invariant state on $B$. Then

(1). $\alpha$ lifts to a coaction $\tilde{\alpha}$ of the Hopf von Neumann algebra $\mathfrak{A}=$ $\pi_{h}(A)^{\prime \prime}$ on the von Neumann algebra $\mathfrak{B}=\pi_{\tau}(B)^{\prime \prime}$ defined by

$$
\tilde{\alpha}\left(\pi_{\tau}(b)\right)=\left(\pi_{\tau} \otimes \pi_{h}\right) \alpha(b), \quad b \in B,
$$

where $\pi_{h}$ and $\pi_{\tau}$ are respectively the GNS representations associated with the Haar state $h$ on $A$ and the state $\tau$ on $B$.

(2). If $\alpha$ is ergodic, then so is $\tilde{\alpha}$.

Proof. (1). We will only show that the natural map $\tilde{\alpha}$ given on the dense subalgebra $\pi_{\tau}(B)$ by

$$
\tilde{\alpha}\left(\pi_{\tau}(b)\right)=\left(\pi_{\tau} \otimes \pi_{h}\right) \alpha(b), \quad b \in B,
$$

is well defined and extends to a normal morphism from $\mathfrak{B}$ to $\mathfrak{B} \otimes \mathfrak{A}$.

Let $b \in B$ and $a \in A$. Denote by $\tilde{b}$ and $\tilde{a}$ respectively the corresponding elements of the Hilbert spaces $H=L^{2}(B, \tau)$ and $K=L^{2}(A, h)$. Define an operator $U$ on $H \otimes K$ by

$$
U(\tilde{b} \otimes \tilde{a})=\left(\pi_{\tau} \otimes \pi_{h}\right) \alpha(b)\left(\tilde{1}_{B} \otimes \tilde{a}\right) .
$$

Then since $\tau$ is $\alpha$ invariant, we have

$$
\begin{aligned}
<U(\tilde{b} \otimes \tilde{a}), U(\tilde{b} \otimes \tilde{a})> & =(\tau \otimes h)\left(1_{B} \otimes a^{*}\right) \alpha\left(b^{*} b\right)\left(1_{B} \otimes a\right) \\
& =a h a^{*}\left(\left(\tau \otimes i d_{A}\right) \alpha\left(b^{*} b\right)\right)=a h a^{*}\left(\tau\left(b^{*} b\right) 1_{A}\right) \\
& =<\tilde{b} \otimes \tilde{a}, \tilde{b} \otimes \tilde{a}>,
\end{aligned}
$$

where $a h a^{*}$ is the functional on $A$ defined by

$$
a h a^{*}(x)=h\left(a^{*} x a\right), \quad x \in A .
$$

Hence $U$ is an isometry. Since $\alpha(B)(1 \otimes A)$ is dense in $B \otimes A, U$ is a unitary operator. We also have

$$
\begin{aligned}
\left(\pi_{\tau} \otimes \pi_{h}\right) \alpha(b) U\left(\tilde{b^{\prime}} \otimes \tilde{a^{\prime}}\right) & =\left(\pi_{\tau} \otimes \pi_{h}\right) \alpha(b)\left(\pi_{\tau} \otimes \pi_{h}\right) \alpha\left(b^{\prime}\right)\left(\tilde{1}_{B} \otimes \tilde{a^{\prime}}\right) \\
& =\left(\pi_{\tau} \otimes \pi_{h}\right) \alpha\left(b b^{\prime}\right)\left(\tilde{1}_{B} \otimes \tilde{a^{\prime}}\right)=U\left(\pi_{\tau}(b) \tilde{b^{\prime}} \otimes \tilde{a^{\prime}}\right) \\
& =U\left(\pi_{\tau}(b) \otimes 1\right)\left(\tilde{b^{\prime}} \otimes \tilde{a^{\prime}}\right) .
\end{aligned}
$$

That is

$$
\left(\pi_{\tau} \otimes \pi_{h}\right) \alpha(b)=U\left(\pi_{\tau}(b) \otimes 1\right) U^{*} .
$$

Condition (1) of Definition 2.4 follows immediately. Since $\alpha(B)(1 \otimes A)$ is dense in $B \otimes A$ (cf. Remark (1) after Definition 2.1] and Podles [23]), Condition (2) of Definition 2.4 follows. 
(2). Assume $\alpha$ is ergodic. Let $z \in \mathfrak{B}$ be a fixed element under $\tilde{\alpha}$ :

$$
\tilde{\alpha}(z)=z \otimes 1_{\mathfrak{A}} .
$$

Let $b_{n} \in B$ be a net of elements such that $\pi_{\tau}\left(b_{n}\right) \rightarrow z$ in the weak operator topology. Consider the average of $\pi_{\tau}\left(b_{n}\right)$ integrated over the quantum group $A$,

$$
z_{n}=\left(i d_{\mathfrak{B}} \otimes h\right) \tilde{\alpha}\left(\pi_{\tau}\left(b_{n}\right)\right),
$$

where we use the same letter $h$ to denote the faithful normal state on $\mathfrak{A}$ determined by the Haar state $h$ on $A$. Then one can verify that $z_{n} \rightarrow z$ in the weak operator topology. Moreover, using

$$
\left(i d_{\mathfrak{A}} \otimes h\right) \Phi(a)=h(a) 1_{\mathfrak{A}}, \quad a \in \mathfrak{A},
$$

where we denote the coproduct on $\mathfrak{A}$ by the same symbol as the coproduct $\Phi$ on $A$, we have

$$
\begin{aligned}
\tilde{\alpha}\left(z_{n}\right) & =\left(i d_{\mathfrak{B}} \otimes i d_{\mathfrak{A}} \otimes h\right)\left(\tilde{\alpha} \otimes i d_{\mathfrak{A}}\right) \tilde{\alpha}\left(\pi_{\tau}\left(b_{n}\right)\right) \\
& =\left(i d_{\mathfrak{B}} \otimes i d_{\mathfrak{A}} \otimes h\right)\left(i d_{\mathfrak{B}} \otimes \Phi\right) \tilde{\alpha}\left(\pi_{\tau}\left(b_{n}\right)\right) \\
& =\left(i d_{\mathfrak{B}} \otimes\left(i d_{\mathfrak{A}} \otimes h\right) \Phi\right) \tilde{\alpha}\left(\pi_{\tau}\left(b_{n}\right)\right) \\
& =\left(i d_{\mathfrak{B}} \otimes h\right) \tilde{\alpha}\left(\pi_{\tau}\left(b_{n}\right)\right) \otimes 1_{\mathfrak{A}}=z_{n} \otimes 1_{\mathfrak{A}} .
\end{aligned}
$$

That is, each $z_{n}$ is fixed under $\tilde{\alpha}$. From part (1) of the theorem, we see

$$
z_{n}=\left(\pi_{\tau} \otimes h\right) \alpha\left(b_{n}\right)=\pi_{\tau}\left(\bar{b}_{n}\right),
$$

where

$$
\bar{b}_{n}=(1 \otimes h) \alpha\left(b_{n}\right) \in B^{\alpha}
$$

is the average of of $b_{n}$. Since $\alpha$ is ergodic, $\bar{b}_{n}$ is a scalar. This implies that each $z_{n}$ is also a scalar. Consequently, the operator $z$, as a limit of the $z_{n}$ 's in the weak operator topology, is a scalar.

Q.E.D.

Remarks. Define on the Hilbert $A$-module $H \otimes A$ (conjugate linear in the second variable) an operator $u$ by

$$
u(\tilde{b} \otimes a)=\left(\pi_{\tau} \otimes 1\right) \alpha(b)\left(\tilde{1}_{B} \otimes a\right) .
$$

Then one verifies that $u$ is a unitary representation of the quantum group $A$ (cf 37, 1, 28]) and $\left(\pi_{\tau}, u\right.$ ) satisfies the following covariance condition in the sense of 0.3 of [1]:

$$
\left(\pi_{\tau} \otimes 1\right) \alpha(b)=u\left(\pi_{\tau}(b) \otimes 1\right) u^{*} .
$$

The operator $U$ defined above is given by

$$
U=\left(1 \otimes \pi_{h}\right) u \text {. }
$$

The pair $\left(\pi_{\tau}, U\right)$ along with the relation (2.7) can be called a covariant system in the framework of Hopf von Neumann algebras. Note that 
part (1) of the above theorem also gives a conceptual proof of Proposition 4.2.(i) of [21], where a rather complicated (and nonconceptual) proof is given.

Notation. Let $v$ be a unitary representation of a quantum group $A$ on some finite dimensional Hilbert space $H_{v}$ [37, 1, 28]. Define

$$
A d_{v}(b)=v(b \otimes 1) v^{*}, \quad b \in B\left(H_{v}\right) .
$$

Then using Proposition 3.2 of [37], we see that $A d_{v}$ is an action of $A$ on $B\left(H_{v}\right)$ (see also the remark after the proof of Theorem 4.1 in [32). It will be called the adjoint action of the quantum group $A$ for the representation $v$. Note that unlike in the case of locally compact groups, for quantum groups we have in general

$$
A d_{v \otimes_{i n} w} \neq A d_{v} \otimes_{i n} A d_{w},
$$

where $\otimes_{i n}$ denotes the interior tensor product representations [37, 29].

For other basic notions on compact quantum groups, we refer the reader to [37, 28, 29].

\section{ERgodic actions of $A_{u}(Q)$ on the Powers factor $R_{\lambda}$} AND THE MurRaY-VON Neumann FACTOR $R$

We construct in this section an ergodic action of the universal quantum group $A_{u}(Q)$ on the type $\mathrm{III}_{\lambda}$ Powers factor $R_{\lambda}$ for a proper choice of $Q$ and an ergodic action of $A_{u}(n)$ on the type $\mathrm{II}_{1}$ Murray-von Neumann factor $R$. These are obtained as consequences of Theorem 3.6 below.

Recall [28, 27, 30] that for every non-singular $n \times n$ complex matrix $Q(n>1$ in the rest of this paper), the universal compact quantum group $\left(A_{u}(Q), u\right)$ is generated by $u_{i j}(i, j=1, \cdots, n)$ with defining relations (with $u=\left(u_{i j}\right)$ ):

$A_{u}(Q): \quad u^{*} u=I_{n}=u u^{*}, \quad u^{t} Q \bar{u} Q^{-1}=I_{n}=Q \bar{u} Q^{-1} u^{t} ;$

There is also another related family of quantum groups $A_{o}(Q)$ [28, 27, 30, 2]:

$$
A_{o}(Q): \quad u^{t} Q u Q^{-1}=I_{n}=Q u Q^{-1} u^{t}, \quad \bar{u}=u ;(\text { here } Q>0)
$$

Part (1) of the next proposition gives a characterization of $A_{u}(Q)$ in terms of the functional $\phi_{Q}$ defined below.

Proposition 3.1. Consider the adjoint action $A d_{u}$ corresponding to the fundamental representation $u$ of the quantum group $\left(A_{u}(Q), u\right)$. 
(1). The quantum group $\left(A_{u}(Q), u\right)$ is the largest compact matrix quantum group such that its action $A d_{u}$ on $M_{n}(\mathbb{C})$ leaves invariant the functional $\phi_{Q}$ defined by

$$
\phi_{Q}(b)=\operatorname{Tr}\left(Q^{t} b\right), \quad b \in M_{n}(\mathbb{C}) .
$$

(2). $A d_{u}$ is an ergodic action if and only if $Q=\lambda E$, where $\lambda$ is a nonzero scalar, $E$ is the positive matrix $(1 \otimes h) u^{t} \bar{u}$ (cf [27]), and $h$ is the Haar measure of $A_{u}(Q)$.

Proof. (1). It is a straightforward calculation to verify that the action $A d_{u}$ of $A_{u}(Q)$ leaves the functional $\phi_{Q}$ invariant.

Assume that $(A, v)$ is a compact quantum group such that $A d_{v}$ leaves $\phi_{Q}$ invariant $\left(v=\left(v_{i j}\right)_{i, j=1}^{n}\right)$. Then the $v_{i j}$ 's satisfy the defining relations for $A_{u}(Q)$. Hence $(A, v)$ is a quantum subgroup of $A_{u}(Q)$.

(2). A matrix $S$ is fixed by $A d_{u}$ if and only if $S$ intertwines the fundamental representation $u$ with itself. Hence the action $A d_{u}$ is ergodic if and only if the fundamental representation $u$ is irreducible. When $Q=\lambda E$, then $A_{u}(Q)=A_{u}(E)$. Since $E$ is positive, $u$ is irreducible (cf. [3]).

On the other hand we have (see [27])

$$
\left(u^{t}\right)^{-1}=E \bar{u} E^{-1},
$$

where $E$ is defined as in the proposition. We also have

$$
\left(u^{t}\right)^{-1}=Q \bar{u} Q^{-1} \text {. }
$$

Hence

$$
E \bar{u} E^{-1}=Q \bar{u} Q^{-1} \text { and } Q^{-1} E \bar{u}=\bar{u} Q^{-1} E .
$$

If $u$ is irreducible, then so is $\bar{u}$ and therefore $Q^{-1} E=$ scalar. Q.E.D.

Note. The proof of necessary condition in (2) above was pointed to us by Woronowicz. Our original proof contains an error.

In general the invariant functional $\phi_{Q}$ defined above is not a trace, even if the action $A d_{u}$ is ergodic. However, for ergodic actions of compact groups on operator algebras, one has the following finiteness theorem of Høegh-Krohn-Landstad-Størmer [15]:

Theorem 3.2. If a von Neumann algebra admits an ergodic action of a compact group $G$, then

(a). this von Neumann algebra is finite;

(b). the unique G-invariant state is a trace on the von Neumann algebra. 
The proposition above shows that part (b) of this finiteness theorem is no longer true for compact quantum groups in general. We now show that part (a) of the above finiteness theorem is false for compact quantum groups either: not only can compact quantum groups act on infinite algebras, they can act on purely infinite factors (type III factors).

Definition 3.3. Let $\left(B_{i}, \pi_{j i}\right)$ be an inductive system of $C^{*}$-algebras $(i, j \in I)$. For each $i \in I$, let $\alpha_{i}$ be an action of a compact quantum group $A$ on $B_{i}$. We say that the actions $\alpha_{i}$ are a compatible system of actions for $\left(B_{i}, \pi_{j i}\right)$ if for each pair $i \leq j$, the following holds,

$$
\left(\pi_{j i} \otimes 1\right) \alpha_{i}=\alpha_{j} \pi_{j i}
$$

The following lemmas will be used in the next theorem. Preserve the notation in Definition 3.3. Let $\pi_{i}$ be the natural embedding of $B_{i}$ into the inductive limit $B$ of the $B_{i}$ 's.

Lemma 3.4. Put for each $i \in I$

$$
\alpha \pi_{i}\left(b_{i}\right)=\left(\pi_{i} \otimes 1\right) \alpha_{i}\left(b_{i}\right), \quad b_{i} \in B_{i} .
$$

Then $\alpha$ induces a well defined action of the quantum group $A$ on $B$. The action $\alpha$ is ergodic if and only if each $\alpha_{i}$ is.

Assume further that $\phi_{i}$ is an inductive system of states on $B_{i}$ and that each $\phi_{i}$ is invariant under $\alpha_{i}$. Then $\alpha$ leaves invariant the inductive limit state $\tau=\lim \phi_{i}$.

Proof. Let $j>i$, so $\pi_{j i}\left(b_{i}\right) \in B_{j}$. Then by the formula of $\alpha$ given in the lemma, we have

$$
\alpha \pi_{j}\left(\pi_{j i}\left(b_{i}\right)\right)=\left(\pi_{j} \otimes 1\right) \alpha_{j}\left(\pi_{j i}\left(b_{i}\right)\right) .
$$

Since $\pi_{j} \pi_{j i}=\pi_{i}$, the left hand of the above is equal to

$$
\alpha \pi_{i}\left(b_{i}\right)=\left(\pi_{i} \otimes 1\right) \alpha_{i}\left(b_{i}\right) .
$$

From the compatibility condition we see that the right hand side of $(*)$ is equal to

$$
\left(\pi_{j} \otimes 1\right)\left(\pi_{j i} \otimes 1\right) \alpha_{i}\left(b_{i}\right)=\left(\pi_{i} \otimes 1\right) \alpha_{i}\left(b_{i}\right) .
$$

This shows that $\alpha$ is well defined on the dense subalgebra $\mathcal{B}=\bigcup \pi_{i}\left(\mathcal{B}_{\rangle}\right)$ of $B$, where $\mathcal{B}_{i}$ is the dense ${ }^{*}$-subalgebra of $B_{i}$ according to Definition 2.1. It also clear that $\alpha$ is bounded and satisfies conditions of Definition 2.1. Hence $\alpha$ induces a well defined action of the quantum group on $B$. 
Assume that each $\alpha_{i}$ is ergodic. It is clear that the action $\alpha$ is ergodic on the dense ${ }^{*}$-subalgebra $\mathcal{B}$. Hence $\alpha$ is ergodic on $B$ by Proposition 2.3.

Conversely, if $\alpha$ is ergodic, then the restrictions $\alpha_{i}$ of $\alpha$ to $B_{i}$ is clearly ergodic.

We now show that $\tau$ is invariant under $\alpha$. Note that $\tau\left(\pi_{i}\left(b_{i}\right)=\phi_{i}\left(b_{i}\right)\right.$. From this we have

$$
\begin{aligned}
(\tau \otimes 1) \alpha\left(\pi_{i}\left(b_{i}\right)\right) & \left.=(\tau \otimes 1)\left(\pi_{i} \otimes 1\right) \alpha_{i}\left(b_{i}\right)\right) \\
& \left.=\left(\phi_{i} \otimes 1\right) \alpha_{i}\left(b_{i}\right)\right)=\phi_{i}\left(b_{i}\right) \\
& =\tau\left(\pi_{i}\left(b_{i}\right)\right) .
\end{aligned}
$$

By density of $\mathcal{B}$ in $B$, we have

$$
(\tau \otimes 1) \alpha(b)=\tau(b), \quad b \in B .
$$

This completes the proof of the lemma.

Q.E.D.

Note. Not every action of a compact quantum group on an inductive limit of $C^{*}$-algebras arises from a compatible system of actions of $A$.

Lemma 3.5. Let $u_{k}$ be a unitary representation of a compact quantum group $A$ on $V_{k}$ for each natural number $k$. Assume that $A d_{u_{k}}$ leaves invariant a functional $\psi_{k}$ on $B\left(V_{k}\right)$. Then the action $A d_{u_{1} \otimes_{i n} \cdots \otimes_{i n} u_{k}}$ leaves the functional $\phi^{k}=\psi_{1} \otimes \cdots \otimes \psi_{k}$ invariant.

Proof. Straightforward calculation.

Q.E.D.

Let $Q \in G L(n, \mathbb{C})$ be a positive matrix with trace 1 . We now construct a sequence of actions $\alpha_{k}$ of the compact quantum group $\left(A_{u}(Q), u\right)$ on $M_{n}(\mathbb{C})^{\otimes k}$. Denote by $u^{k}$ the $k$-th fold interior tensor product of the representation $u$, i.e.,

$$
u^{k}=u \otimes_{i n} \cdots \otimes_{i n} u,
$$

see [29] for the definition of the interior tensor product $\otimes_{i n}$. Put

$$
\alpha_{k}=A d_{u^{k}}, \quad \phi_{Q}^{k}=\phi_{Q}^{\otimes k}=\phi_{Q} \otimes \cdots \otimes \phi_{Q}
$$

Let

$$
\begin{gathered}
B=\lim _{k \rightarrow \infty} M_{n}(\mathbb{C})^{\otimes k}, \quad \tau_{Q}=\lim _{k \rightarrow \infty} \phi_{Q}^{k}, \\
\mathfrak{B}=\pi_{Q}(B)^{\prime \prime}, \quad \mathfrak{A}=\pi_{h}\left(A_{u}(Q)\right)^{\prime \prime},
\end{gathered}
$$

where $\pi_{Q}$ and $\pi_{h}$ are respectively the GNS-representations for the positive functional $\tau_{Q}$ and the Haar state $h$ on $A_{u}(Q)$. 
Theorem 3.6. The actions $\alpha_{k}(k=1,2, \cdots)$ of $A_{u}(Q)$ forms a compatible system of ergodic actions leaving the functionals $\phi_{Q}^{k}$ invariant. These actions gives rise to a natural ergodic action on the UHF algebra $B$ leaving invariant the positive functional $\tau_{Q}$, which in turn lifts to an ergodic action on the von Neumann algebra $\mathfrak{B}$.

Proof. It is straightforward to verify that the actions $\alpha_{k}$ are a compatible system of actions. Since each $u^{k}$ is irreducible (cf [3]), we see that the actions $\alpha_{k}$ are ergodic. By 3.1.(1), $\phi_{Q}$ is invariant under the action $A d_{u}$. Hence applying the lemmas above, we see that the functionals $\phi_{Q}^{k}$ are invariant under the actions $\alpha_{k}$, and these actions gives rise to an ergodic action of the quantum group $A$ on $B$ leaving $\tau_{Q}$ invariant.

Now apply Theorem 2.5, the action $\alpha$ on $B$ induces an ergodic action

$$
\tilde{\alpha}: \mathfrak{B} \longrightarrow \mathfrak{B} \otimes \mathfrak{A}
$$

at the von Neumann algebra level defined by

$$
\tilde{\alpha}\left(\pi_{Q}(b)\right)=\left(\pi_{Q} \otimes \pi_{h}\right) \alpha(b),
$$

where $b \in B$.

Q.E.D.

Corollary 3.7. Take

$$
Q=\left(\begin{array}{cc}
a & 0 \\
0 & 1-a
\end{array}\right), \quad a \in(0,1 / 2) .
$$

Then $\tau_{Q}$ is the Powers state, so the quantum group $A_{u}(Q)$ acts ergodically on the Powers factor $R_{\lambda}$ of type $\mathrm{III}_{\lambda}$, where $\lambda=a /(1-a)$.

Corollary 3.8. (compare [34]) Take $Q=I_{n}$. Then $\tau_{Q}$ is the unique trace on the UHF algebra $B$ of type $n^{\infty}$, so the quantum group $A_{u}(n)=$ $A_{u}\left(I_{n}\right)$ acts ergodically on the hyperfinite $\mathrm{II}_{1}$ factor $R$.

We will see in $\$ 5$ that for appropriate choice of $Q$, the quantum groups $A_{u}(Q)$ act on the injective factor $R_{\infty}$ of type $\mathrm{III}_{1}$ also. It would be interesting to know whether compact quantum groups admit ergodic actions on factors of type $\mathrm{III}_{0}$ too.

\section{FIXED POINT SUbALGEBRAS OF QUANTUM SUbGROUPS}

In this section, we show that although the actions of the universal quantum groups $A_{u}(Q)$ constructed in the last section are ergodic, when restricted to some of their non-trivial quantum subgroups, we obtain interesting large fixed point algebras.

Let $Q=\left(\begin{array}{cc}a & 0 \\ 0 & 1-a\end{array}\right)$, as in 3.7. Put $q=\lambda^{1 / 2}=(a /(1-a))^{1 / 2}$. Then from the definitions of $S U_{q}(2)$ and $A_{u}(Q)$, we see that $S U_{q}(2)$ 
is a quantum subgroup of $A_{u}(Q)$. By restriction, we obtain from the action of $A_{u}(Q)$ an action of $S U_{q}(2)$ on $R_{\lambda}$. The fixed point subalgebra of $R_{\lambda}$ under the action of $S U_{q}(2)$ is generated by the Jones projections $\left\{1, e_{1}, e_{2}, \cdots,\right\}$. The restriction of the Powers states $\tau_{Q}$ to this fixed point algebra is a trace and its values on the Jones projections gives the Jones polynomial. See the book of Jones [18].

Now take $Q=\frac{1}{n} I_{n}$. We have Corollary 3.8. For simplicity of notation, let $\tau$ denote the trace $\tau_{Q}$ on the UHF algebra $B$. There are two special quantum subgroups of $A_{u}(n): S U(n)$ and $A_{o}(Q)=A_{o}(n)$. By 4.7.d. of [14], for any closed subgroup $G$ of $S U(n)$, the fixed point algebra $R^{G}$ is a $\mathrm{II}_{1}$ subfactor of $R$. We now show that the same result holds for quantum subgroups of $A_{o}(n)$. For this, it suffices to prove the following

Proposition 4.1. The fixed point subalgebra $R^{A_{o}(n)}$ of $R$ for the quantum subgroup $A_{o}(n)$ of $A_{u}(n)$ is a $\mathrm{II}_{1}$ factor and the action of $A_{o}(n)$ on $R$ is prime.

Proof. Put $\beta=n^{2}$. By [2], the fixed point subalgebra of $M_{n}(\mathbb{C})^{\otimes k}$ for the action

$$
\alpha_{k}=A d_{u^{k}}
$$

is generated by $1, e_{1}, \cdots, e_{k-1}$, where $u$ is the fundamental representation of the quantum group $A_{o}(n)$,

$$
e_{s}=I_{H^{\otimes(s-1)}} \otimes \sum_{i, j} \frac{1}{n} e_{i j} \otimes e_{i j} \otimes I_{H^{\otimes(k-s-1)}},
$$

and $H=\mathbb{C}^{n}$. The e's satisfies the relations:

(i). $e_{s}^{2}=e_{s}=e_{s}^{*}$;

(ii). $e_{s} e_{t}=e_{t} e_{s}, 1 \leq s, t \leq k-1,|s-t| \geq 2$;

(iii). $\beta e_{s} e_{t} e_{s}=e_{s}, 1 \leq s, t \leq k-1,|s-t|=1$.

We now show that the restriction of $\tau$ on the fixed point subalgebra of $M_{n}(\mathbb{C})^{\otimes k}$ satisfies the Markov trace condition of modulus $\beta$, where $\tau$ is the trace on $R$. Namely, we will verify the identity

$$
\tau\left(w e_{k-1}\right)=\frac{1}{\beta} \tau(w)
$$

for $w$ in the subalgebra of $M_{n}(\mathbb{C})^{\otimes k}$ generated by $1, e_{1}, \cdots, e_{k-2}$. By Theorem 4.1.1 and Corollary 2.2.4 of Jones [17], this will complete the proof of the proposition. It will also follow that the action of $A_{o}(n)$ on $R$ is prime. To verify this, it suffices by Proposition 2.8.1 of [14 to check the Markov trace condition for $w$ of the form

$$
w=\left(e_{i_{1}} e_{i_{1}-1} \cdots e_{j_{1}}\right)\left(e_{i_{2}} e_{i_{2}-1} \cdots e_{j_{2}}\right) \cdots\left(e_{i_{p}} e_{i_{p}-1} \cdots e_{j_{p}}\right),
$$


where

$$
\begin{aligned}
& 1 \leq i_{1}<i_{2} \cdots i_{p} \leq k-2 \\
& 1 \leq j_{1}<j_{2} \cdots j_{p} \leq k-2 \\
& i_{1} \geq j_{1}, i_{2} \geq j_{2}, \cdots, i_{p} \geq j_{p} \\
& 0 \leq p \leq k-2
\end{aligned}
$$

If $i_{p}<k-2$ then it is easy to see that

$$
\tau\left(w e_{k-1}\right)=\tau(w) \tau\left(e_{k-1}\right)=\frac{1}{\beta} \tau(w),
$$

noting that $\tau\left(e_{k-1}\right)=\frac{1}{\beta}$. Hence we can assume $i_{p}=k-2$. Let $l_{w}$ be the length of the word $w$. Then $w$ takes the form

$$
w=\sum\left(\frac{1}{n}\right)^{l_{w}}(\cdots) \otimes e_{a b} \otimes 1,
$$

where the summation is over the indices $a, b$ and some other indices that need not to be specified, and the terms in $(\cdots)$ are certain elements of $M_{n}(\mathbb{C})^{\otimes(k-2)}$ that need not to be specified either (the components in the tensor product of the terms in $(\cdots)$ are products of $e_{i j}$ 's). We have then

$$
\begin{aligned}
\tau\left(w e_{k-1}\right) & =\left(\frac{1}{n}\right)^{l_{w}+1} \sum_{x, y} \sum \tau\left((\cdots) \otimes e_{a b} e_{x y} \otimes e_{x y}\right) \\
& =\left(\frac{1}{n}\right)^{l_{w}+1} \sum_{x, y} \sum \tau\left((\cdots) \otimes e_{a b} e_{x y} \otimes 1\right) \tau\left(I_{H^{\otimes(k-1)}} \otimes e_{x y}\right) \\
& =\left(\frac{1}{n}\right)^{l_{w}+1} \tau\left(\sum_{x} \sum\left((\cdots) \otimes e_{a b} e_{x x} \otimes 1\right) \frac{1}{n}\right) \\
& =\frac{1}{\beta} \tau(w)
\end{aligned}
$$

The proof is complete.

Q.E.D.

Remarks. (1). In view of the above result, fixed point algebras of quantum subgroups of $A_{o}(n)$ give examples of subfactors. Therefore, it would be interesting to classify finite quantum subgroups of $A_{o}(n)$ and study them in the light of Jones' theory, see [14] for this in the case of the Lie group $S U(2)$. Note that the quantum $A_{o}(n)$ contains the quantum permutation group $A_{\text {aut }}\left(X_{n}\right)$ of $n$ point space $X_{n}$ (see [32]) and many other interesting quantum subgroups (see [28]). It would also be interesting to determine the fixed point subalgebras of the quantum subgroups of $S U_{-1}(n)\left(S U_{-1}(n)\right.$ is a quantum subgroup of 
$A_{u}(n)$ because its antipode has period 2 [28, 27]). We refer the reader to Banica [4] for some interesting related results.

(2). Note that since

$$
A d_{v \otimes_{i n} w} \neq A d_{v} \otimes_{i n} A d_{w}
$$

for unitary representation $v$ and $w$ of $A_{o}(n)$, we do not have a commuting square like the one on p222 of [14 for a given quantum subgroup $G$ of $A_{o}(n)$.

\section{Ergodic actions of $A_{u}(Q)$ on the Cuntz algebra And THE INJECTIVE FACTOR OF TYPE III $_{1}$}

The Cuntz algebra $\mathcal{O}_{n}$ is an infinite simple $C^{*}$-algebra without trace, hence by 15 it does not admit an ergodic action of a compact group. Recall that the Cuntz algebra $\mathcal{O}_{n}$ is the simple $C^{*}$-algebra generated by $n$ isometries $S_{k}(k=1, \cdots, n)$ such that

$$
\sum_{k=1}^{n} S_{k} S_{k}^{*}=1 .
$$

Just as $U(n)$, the compact matrix quantum group $A_{u}(Q)$ acts on $\mathcal{O}_{n}$ in a natural manner [12, 10, 19], where $Q$ is a positive matrix of trace 1 in $G L(n, \mathbb{C})$ :

$$
\alpha\left(S_{j}\right)=\sum_{i=1}^{n} S_{i} \otimes u_{i j},
$$

the dense ${ }^{*}$-algebra $\mathcal{B}$ of Definition 2.1 being the ${ }^{*}$-subalgebra ${ }^{0} \mathcal{O}_{n}$ of $\mathcal{O}_{n}$ generated by the $S_{i}$ 's, see Doplicher-Roberts [11]. However, unlike the actions of compact groups on $\mathcal{O}_{n}$, we have

Theorem 5.1. The above action $\alpha$ of the quantum group $A_{u}(Q)$ on $\mathcal{O}_{n}$ is ergodic, the unique $\alpha$-invariant state on $\mathcal{O}_{n}$ is the quasi-free state $\omega_{Q}$ associated with $Q$ [13.

Proof. Let $H$ be the Hilbert subspace of $\mathcal{O}_{n}$ linearly spanned by the $S_{k}$ 's. Let $\left(H^{s}, H^{r}\right)$ be the linear span of elements of the form $S_{i_{1}} S_{i_{2}} \cdots S_{i_{r}} S_{j_{s}}^{*} \cdots S_{j_{2}}^{*} S_{j_{1}}^{*}$. Then ${ }^{0} \mathcal{O}_{n}$ is the linear span of all the spaces $\left(H^{s}, H^{r}\right), r, s \geq 0$ (see [11]). Observe that each of the spaces $\left(H^{s}, H^{r}\right)$ is invariant under the action $\alpha$ :

$$
\begin{gathered}
\alpha\left(S_{i_{1}} S_{i_{2}} \cdots S_{i_{r}} S_{j_{s}}^{*} \cdots S_{j_{2}}^{*} S_{j_{1}}^{*}\right)= \\
\sum_{k_{1}, \cdots k_{r}, l_{1}, \cdots, l_{s}=1}^{n} S_{k_{1}} S_{k_{2}} \cdots S_{k_{r}} S_{l_{s}}^{*} \cdots S_{l_{2}}^{*} S_{l_{1}}^{*} \otimes u_{k_{1} i_{1}} u_{k_{2} i_{2}} \cdots u_{k_{r} i_{r}} u_{l_{s} j_{s}}^{*} \cdots u_{l_{2} j_{2}}^{*} u_{l_{1} j_{1}}^{*}
\end{gathered}
$$


Hence $(i d \otimes h) \alpha\left(\left(H^{s}, H^{r}\right)\right)$ is the space of the fixed elements of $\left(H^{s}, H^{r}\right)$ under $\alpha$, where $h$ is the Haar state on $A_{u}(Q)$. For $r \neq s$, the tensor product representations $u^{\otimes r}$ and $u^{\otimes s}$ of the fundamental representation $u$ of the quantum group $A_{u}(Q)$ are inequivalent and irreducible [3]. Hence by Theorem 5.7 of Woronowicz [37], for $r \neq s$,

$$
h\left(u_{k_{1} i_{1}} u_{k_{2} i_{2}} \cdots u_{k_{r} i_{r}} u_{l_{s} j_{s}}^{*} \cdots u_{l_{2} j_{2}}^{*} u_{l_{1} j_{1}}^{*}\right)=0,
$$

and therefore $\left(H^{s}, H^{r}\right)$ has no fixed point other than 0 . For $r=s$, identifying the elements

$$
S_{i_{1}} S_{i_{2}} \cdots S_{i_{r}} S_{j_{r}}^{*} \cdots S_{j_{2}}^{*} S_{j_{1}}^{*}
$$

of $\left(H^{r}, H^{r}\right)$ with the matrix units

$$
e_{i_{1} j_{1}} \otimes e_{i_{2} j_{2}} \otimes \cdots \otimes e_{i_{r} j_{r}}
$$

of $M_{n}(\mathbb{C})^{\otimes r}$, the action $\alpha$ on $\left(H^{r}, H^{r}\right)$ is identified with the action $\alpha_{r}$ on $M_{n}(\mathbb{C})^{\otimes r}$ of Theorem 3.6. Hence the fixed elements of $\left(H^{r}, H^{r}\right)$ under $\alpha$ are the scalars. Consequently, the fixed elements of ${ }^{0} \mathcal{O}_{n}$ under $\alpha$ are the scalars. By Proposition 2.3, $\alpha$ is ergodic on $\mathcal{O}_{n}$.

Let $\phi$ be the (unique) $\alpha$-invariant state on $\mathcal{O}_{n}$. Then for $x \in\left(H^{r}, H^{s}\right)$ with $r \neq s, r, s \geq 0$, we have

$$
\phi(x)=h((\phi \otimes 1) \alpha(x))=\phi((1 \otimes h) \alpha(x)) .
$$

But $(1 \otimes h) \alpha(x)=0$ according to the computation above. Hence $\phi(x)=0$. From the consideration of the last paragraph, $\alpha$ restricts to an ergodic action on the subalgebra $\left(H^{k}, H^{k}\right)$ of $\mathcal{O}_{n}$. Identifying $\left(H^{k}, H^{k}\right)$ with $M_{n}(\mathbb{C})^{\otimes k}$ as above, we see that

$$
\phi(x)=\phi_{Q}^{k}(x), \quad x \in\left(H^{k}, H^{k}\right),
$$

where $\phi_{Q}^{k}$ is the functional in Theorem 3.6. This shows that $\phi$ is the quasi-trace state $\omega_{Q}$ associated with $Q(\operatorname{cf}[13])$.

Q.E.D.

We can assume that $Q=\operatorname{diag}\left(q_{1}, q_{2}, \cdots, q_{n}\right)$ is a diagonal positive matrix with trace 1 , since $A_{u}(Q)$ and $A_{u}\left(V Q V^{-1}\right)$ are similar to each other [27]. Let $\beta$ be a positive number. Define numbers $\omega_{1}, \omega_{2}, \cdots, \omega_{n}$ by

$$
\operatorname{diag}\left(e^{-\beta \omega_{1}}, e^{-\beta \omega_{2}}, e^{-\beta \omega_{n}}\right)=\operatorname{diag}\left(q_{1}, q_{2}, \cdots, q_{n}\right) .
$$

Let $\pi_{Q}$ be the GNS representation of the $\alpha$-invariant state $\omega_{Q}$ of $\mathcal{O}_{n}$. Then by Theorem 4.7 of Izumi [16] and Theorem 2.5, we have

Corollary 5.2. If $\omega_{1} / \omega_{k}$ is irrational for some $k$, then the compact quantum group $A_{u}(Q)$ acts ergodically on the injective factor $\pi_{Q}\left(\mathcal{O}_{n}\right)^{\prime \prime}$ of type $\mathrm{III}_{1}$. 
Remarks. (1). The big quantum semi-group $U_{n c}(n)$ of Brown also acts on $\mathcal{O}_{n}$ in the same way as $A_{u}(Q)$ on $\mathcal{O}_{n}$ above. See Brown [6] and 4.1 of Wang 28] for the quantum semi-group structure on $U_{n c}(n)$.

(2). If the $\omega_{1} / \omega_{k}$ 's are rational for all $k$, then $\pi_{Q}\left(\mathcal{O}_{n}\right)^{\prime \prime}$ is an injective factor of type $\mathrm{III}_{\lambda}$, on which $A_{u}(Q)$ acts ergodically, where $\lambda$ is determined from an equation involving $q_{1}, \cdots, q_{n}$ (see [16]). In particular, taking $A_{u}(Q)=A_{u}(n)$, we see that even the compact matrix quantum group $A_{u}(n)$ of Kac type admits ergodic actions on both the infinite $C^{*}$-algebra $\mathcal{O}_{n}$ and the injective factor $\pi_{Q}\left(\mathcal{O}_{n}\right)^{\prime \prime}$ of type $\operatorname{III}_{\frac{1}{n}}$. In view of Corollary 3.8, it would be interesting to solve the following problem:

Problem: Does a compact matrix quantum group of non-Kac type admit ergodic action on the hyperfinite $\mathrm{II}_{1}$ factor $R$ ?

\section{ERgodic ACtIONS ON QUOTIENT SPACES}

In this section, we study ergodic actions of compact quantum groups on their quantum quotient spaces. We also give an example to show that, contrary to the classical situation, not all ergodic actions arise in this way.

Fix a quantum subgroup $H$ of a compact quantum group $G$, which is given by a surjective morphism $\theta$ of Woronowicz Hopf $C^{*}$-algebras from $C(G)$ to $C(H)$. Let $h_{H}$ and $h_{G}$ be respectively the Haar states on $C(H)$ and $C(G)$. Then there is a natural action $\beta$ of the quantum group $H$ on $G$ given by

$$
\beta: C(G) \longrightarrow C(H) \otimes C(G), \quad \beta=(\theta \otimes 1) \Phi_{G},
$$

where $\Phi_{G}$ is the coproduct on $C(G)$. The quotient space $H \backslash G$ is defined by the fixed point algebra of $\beta$ (cf [23):

$$
C(H \backslash G)=C(G)^{\beta}=\left\{a \in C(G):(\theta \otimes 1) \Phi_{G}(a)=1 \otimes a\right\} .
$$

The restriction of $\Phi_{G}$ to $C(H \backslash G)$ defines a natural action $\alpha$ of $G$ on $C(H \backslash G)$ :

$$
\alpha=\left.\Phi_{G}\right|_{C(H \backslash G)}: C(H \backslash G) \longrightarrow C(H \backslash G) \otimes C(G) .
$$

The dense ${ }^{*}$-subalgebras of Definition 2.1 for the actions $\beta$ and $\alpha$ are the natural ones. Note that $E=\left(h_{H} \otimes 1\right) \beta=\left(h_{H} \theta \otimes 1\right) \Phi_{G}$ is a projection of norm one from $C(G)$ to $C(H \backslash G$ ) (cf Proposition 2.3 and [23).

Proposition 6.1. In the situation as above, we have

(1). the action $\alpha$ of $G$ on $C(H \backslash G)$ is ergodic; 
(2). $C(H \backslash G)$ has a unique $\alpha$ invariant state $\omega$ satisfying

$$
h_{G}(a)=\omega\left(\left(h_{H} \theta \otimes 1\right) \Phi_{G}(a)\right), \quad a \in C(G) .
$$

Namely, $\omega$ is the restriction of $h_{G}$ on $C(H \backslash G)$.

Note. Part (2) of the proposition above is the analogue of the the following well known integration formula in the classical situation:

$$
\int_{G} a(g) d g=\int_{H \backslash G} \int_{H} a(h g) d h d \omega(g), \quad a \in C(G) .
$$

Proof. (1). Let $a \in C(H \backslash G)$ be fixed under $\alpha$, i.e.,

$$
\alpha(a)=a \otimes 1 \text {. }
$$

Since $\alpha(a)=\Phi_{G}(a)$ and since (by the definition of $C(H \backslash G)$ )

$$
(\theta \otimes 1) \Phi_{G}(a)=1 \otimes a,
$$

it follows that

$$
(\theta \otimes 1) \alpha(a)=1 \otimes a .
$$

Using $(* *)$ for the left hand side of the above, we get

$$
\theta(a) \otimes 1=1 \otimes a .
$$

This is possible only for $a=\lambda \cdot 1$ for some scalar $\lambda$.

(2). The general result of the existence and uniqueness of the invariant state for an ergodic action is proven in [5]. For the special situation we consider here, we now not only prove the existence and uniqueness of the invariant state, but also give the precise formula of the invariant state.

Let $\omega$ be the restriction of $h_{G}$ on the subalgebra $C(H \backslash G)$ of $C(G)$. Since $\left(h_{H} \theta \otimes 1\right) \Phi_{G}$ is a projection from $C(G)$ onto $C(H \backslash G)$ and $\alpha$ is the restriction of $\Phi_{G}$ on $C(H \backslash G)$, the invariance of $\omega$ for the action $\alpha$ follows from the invariance of the Haar state $h_{G}$.

Conversely, let $\mu$ be any invariant state on $C(H \backslash G)$. Using again the fact that $\left(h_{H} \theta \otimes 1\right) \Phi_{G}$ is a projection from $C(G)$ onto $C(H \backslash G)$, a standard calculation shows that the functional

$$
\phi(a)=\mu\left(\left(h_{H} \theta \otimes 1\right) \Phi_{G}(a)\right), \quad a \in C(G)
$$

is a right invariant state, i.e.

$$
\phi * \psi(a)=\phi(a), \quad a \in C(G),
$$

where $\psi$ is a state on $A$ and $\phi * \psi=(\phi \otimes \psi) \Phi_{G}$ is the convolution operation (cf [37]). From the uniqueness of the Haar state, it follows from this that

$$
\phi=h_{G}, \quad \mu=\omega=\left.h_{G}\right|_{C(H \backslash G)} .
$$


Q.E.D.

Remarks. (1). Note that the quantum groups $A_{u}(Q), A_{o}(Q)$ and $B_{u}(Q)$ have many quantum subgroups. In the light of Proposition 6.1 and Theorem 2.5, it would be interesting to study the corresponding operator algebras and the actions on them. We leave this to a separate work.

(2). More general than the considerations in Proposition 6.1, if two quantum groups admit commuting actions on a noncommutative space, then they act on each other's orbit spaces (not necessarily in an ergodic manner), just as in the classical situation. Note that the notion of orbit space corresponds to fixed point algebra in the noncommutative situation.

An Example. Every transitive action of a compact group $G$ on a topological space $X$ is isomorphic to the natural action of $G$ on $H \backslash G$, where $H$ is the closed subgroup of $G$ that fixes some point of $X$. However, this is no longer true for quantum groups, even if the space on which the quantum group acts is a classical one.

To see this, let $X_{n}=\left\{x_{1}, \cdots, x_{n}\right\}$ be the space with $n$ points. By Theorem 3.1 of [32], the quantum automorphism group $A_{\text {aut }}\left(X_{4}\right)$ of $X_{4}$ contains the ordinary permutation group $S_{4}$, hence it acts ergodically on $X_{4}$. The quantum subgroup of $A_{a u t}\left(X_{4}\right)$ that fix a point, say $x_{1}$, is isomorphic to $A_{\text {aut }}\left(X_{3}\right)$, which is the same as $C\left(S_{3}\right)$, a (commutative) algebra of dimension 6. From [32], we know that as a $C^{*}$-algebra, $A_{\text {aut }}\left(X_{n}\right)$ is the same as $C\left(S_{n}\right)$ for $n \leq 3$ and it has $C^{*}(\mathbb{Z} / 2 \mathbb{Z} * \mathbb{Z} / 2 \mathbb{Z})$ as a quotient for $n \geq 4$, where $\mathbb{Z} / 2 \mathbb{Z} * \mathbb{Z} / 2 \mathbb{Z}$ is the free product of the two-element group $\mathbb{Z} / 2 \mathbb{Z}$ with itself, because the entries of the matrix

$$
\left(\begin{array}{cccc}
p & 1-p & 0 & 0 \\
1-p & p & 0 & 0 \\
0 & 0 & q & 1-q \\
0 & 0 & 1-q & q
\end{array}\right)
$$

satisfy the commutation relations of the algebra $A_{\text {aut }}\left(X_{4}\right)$, where $p, q$ are the projections generating the $C^{*}$-algebra $C^{*}(\mathbb{Z} / 2 \mathbb{Z} * \mathbb{Z} / 2 \mathbb{Z}): p=$ $(1-u) / 2$ and $q=(1-v) / 2, u$ and $v$ being the unitary generators of the first and second copies of $\mathbb{Z} / 2 \mathbb{Z}$ in the free product (cf [24]).

For simplicity of notation, let $C(G)=A_{\text {aut }}\left(X_{4}\right)$, and let $\mathcal{M}$ be the canonical dense subalgebra of $C(G)$ generated by the coefficients of the fundamental representation of $G$ (see [37]). Let $H=S_{3}$, the subgroup of $G$ that fixes $x_{1}$, and let $\mathcal{H}=C(H)$. Let $\theta$ be the surjection from $C(G)$ to $C(H)$ that embeds $H$ as subgroup of $G$ (cf. [32]). Let $\beta$ be 
the action defined in the beginning of this section. We claim that the coset space $H \backslash G$ is not isomorphic to $X_{4}$ as a $G$-space (see Sect. 2 of 32 for the notion of morphism). Namely, we have

Proposition 6.2. The G-algebras $C(H \backslash G)$ (which is defined to be $\left.C(G)^{\beta}\right)$ and $C\left(X_{4}\right)$ are not isomorphic to each other.

Proof. Since $C\left(X_{4}\right)$ has dimension 4 , it suffices to show that $C(H \backslash G)$ is infinite dimensional.

We make $\mathcal{M}$ into a Hopf $\mathcal{H}$-module (i.e. a compatible system of a left $\mathcal{H}$ comodule and a left $\mathcal{H}$ module) as follows. The restriction of $\beta$ to $\mathcal{M}$ clearly defines a left $\mathcal{H}$ comodule structure:

$$
\beta: \mathcal{M} \longrightarrow \mathcal{H} \otimes \mathcal{M} \text {. }
$$

The left $\mathcal{H}$ module structure on $\mathcal{M}$ is the trivial one defined by

$$
\begin{aligned}
& \mathcal{H} \otimes \mathcal{M} \longrightarrow \mathcal{M}, \\
& h \cdot m=\epsilon(h) m, \quad h \in \mathcal{H}, m \in \mathcal{M} .
\end{aligned}
$$

By Theorem 4.1.1 of Sweedler [25], we have an isomorphism of left $\mathcal{H}$ modules

$$
\mathcal{H} \otimes \mathcal{M}^{\beta} \cong \mathcal{M}
$$

That is

$$
\begin{aligned}
& \mathcal{H} \otimes \mathcal{A}(H \backslash G) \cong \mathcal{M} \\
& h \otimes m^{\prime} \mapsto h \cdot m^{\prime}, \quad h \in \mathcal{H}, m^{\prime} \in \mathcal{A}(H \backslash G),
\end{aligned}
$$

where $\mathcal{A}(H \backslash G)=\mathcal{M}^{\beta}$ is the canonical dense subalgebra of $C(H \backslash G)=$ $C(G)^{\beta}$. Since $\mathcal{M}$ is infinite dimensional and $\mathcal{H}$ is finite dimensional, $\mathcal{A}(H \backslash G)$ and therefore $C(H \backslash G)$ are also infinite dimensional. Q.E.D.

Acknowledgement. The author is indebted to Marc A. Rieffel for continual support. Part of this paper was written while the author was a member at the IHES during the year July, 1995-Aug, 1996. He thanks the IHES for its financial support and hospitality during this period. The author also wishes to thank the Department of Mathematics at UC-Berkeley for its support and hospitality while the author holds an NSF Postdoctoral Fellowship there during the final stage of this paper.

\section{REFERENCES}

[1] Baaj, S. and Skandalis, G.: Unitaires multiplicatifs et dualité pour les produits croisés de $C^{*}$-algèbres, Ann. Sci. Ec. Norm. Sup. 26 (1993), 425-488.

[2] Banica, T.: Théorie des représentations du groupe quantique compact libre O(n), C. R. Acad. Sci. Paris t. 322, Serie I (1996), 241-244. 
[3] Banica, T.: Le groupe quantique compact libre $U(n)$, Commun. Math. Phys. 190 (1997), 143-172.

[4] Banica, T.: Quantum groups acting on $n$ points, complex Hadamard matrix and a construction of subfactors, math/9806054

[5] Boca, F.: Ergodic actions of compact matrix pseudogroups on $C^{*}$-algebras, in Recent Advances in Operator Algebras, Astérisque 232 (1995), 93-109.

[6] Brown, L.: Ext of certain free product of $C^{*}$-algebras, J. Operator Theory 6 (1981), 135-141.

[7] Ceccherini, T., Doplicher, S., Pinzari, C. and Roberts, J.E.: A generalization of the Cuntz algebras and model actions, J. Funct. Anal. 125 (1994), 416-437.

[8] Cuntz, Joachim: Simple $C^{*}$-algebras generated by isometries, Comm. Math. Phys. 57 (1977), no. 2, 173-185.

[9] Cuntz, Joachim: Regular actions of Hopf algebras on the $C^{*}$-algebra generated by a Hilbert space, in Operator algebras, mathematical physics, and low-dimensional topology(Istanbul, 1991), 87-100, A K Peters, Wellesley, MA, 1993; MR 94m:461

[10] Doplicher, S.: Abstract compact group duals, operator algebras and quantum field theory, Proc. ICM-1990, Kyoto, Springer, 1991.

[11] Doplicher, S. and Roberts, J.E.: Duals of compact Lie groups realized in the Cuntz algebras and their actions on $C^{*}$-algebras, J. Funct. Anal. 74 (1987), 96-120.

[12] Doplicher, S. and Roberts, J.E.: Compact group actions on $C^{*}$-algebras, $J$. Operator Theory 19 (1988), 283-305.

[13] Evans, David E.: On $\mathcal{O}_{n}$, Publ. RIMS. Kyoto Univ. 16 (1980), 915-927.

[14] Goodman, F.M. and de la Harpe, P. and Jones, V.F.R.: Coxeter Graphs and Towers of Algebras, MSRI Publ. 14, Springer-Verlag, 1989.

[15] Høegh-Krohn, R. and Lanstad, M.B. and Størmer, E.: Compact ergodic groups of automorphisms, Ann. of Math. 114 (1981), 75-86

[16] Izumi, Masaki: Subalgebras of infinite $C^{*}$-algebras with finite Watatani indices. I. Cuntz algebras, Comm. Math. Phys. 155 (1993), no. 1, 157-182; MR 94e:46104

[17] Jones, V. F. R.: Index for Subfactors, Invent. Math. 72 (1983), 1-25.

[18] Jones, V. F. R.: Subfactors and Knots, Regional Conference Series 80, Amer. Math. Soc., 1991.

[19] Konishi, Y., Nagisa, M. and Watatani, Y.: Some remarks on actions of compact matrix quantum groups on $C^{*}$-algebras, Pacific J. Math. 153 (1992), 119-127.

[20] Marciniak, M.: Actions of compact quantum groups on $C^{*}$-algebras, Proc. AMS 126 (1998), 607-616.

[21] Nakagami, Y.: Takesaki duality for the crossed product by quantum groups, in Quantum and Non-Commutative Analysis, H. Araki ed., Kluwer Academic Publishers, 1993, 263-281.

[22] Paolucci, A.: Coactions of Hopf algebras on Cuntz algebras and their fixed point algebras, Proc. AMS 125 (1997), 1033-1042.

[23] Podles, P.: Symmetries of quantum spaces. Subgroups and quotient spaces of quantum $S U(2)$ and $S O(3)$ groups, Commun. Math. Phys. 170 (1995), 1-20.

[24] Raeburn, I. and Sinclair, A.M.: The $C^{*}$-algebra generated by two projections, Math. Scand. 65 (1989), 278-290.

[25] Sweedler, M.E.: Hopf Algebras, Benjamin, New York, 1969. 
[26] Van Daele, A.: The Haar measure on a compact quantum group, Proc. Amer. Math. Soc. 123 (1995), 3125-3128.

[27] Van Daele, A. and Wang, S. Z.: Universal quantum groups, International J. Math 7:2 (1996), 255-264.

[28] Wang, S. Z.: Free products of compact quantum groups, Commun. Math. Phys. 167 (1995), 671-692.

[29] Wang, S. Z.: Tensor products and crossed products of compact quantum groups, Proc. London Math. Soc. 71 (1995), 695-720.

[30] Wang, S. Z.: New classes of compact quantum groups, Lecture notes for talks at the University of Amsterdam and the University of Warsaw, January and March, 1995.

[31] Wang, S. Z.: Problems in the theory of quantum groups, in Quantum Groups and Quantum Spaces, Banach Center Publication 40 (1997), Inst. of Math., Polish Acad. Sci., Editors: R. Budzynski, W. Pusz, and S. Zakrzewski. pp6778

[32] Wang, S. Z.: Quantum symmetry groups of finite spaces, Commun. Math. Phys. 195:1 (1998), 195-211.

[33] Wassermann, A.: Ergodic actions of compact groups on operator algebras I: General theory, Ann. of Math. 130 (1989), 273-319.

[34] Wassermann, A.: Ergodic actions of compact groups on operator algebras III: Classification for SU(2), Invent. Math. 93 (1988), 309-355.

[35] Wassermann, A.: Coactions and Yang-Baxter equations for ergodic actions and subfactors, in Operator Algebras and Applications, no 2, ed. by D. Eavans and M. Takesaki, London Math. Soc. Lecture Notes 136 (1988), 203-236.

[36] Woronowicz, S. L.: Twisted $S U(2)$ group. An example of noncommutative differential calculus, Publ. RIMS, Kyoto Univ. 23 (1987), 117-181.

[37] Woronowicz, S. L.: Compact matrix pseudogroups, Commun. Math. Phys. 111 (1987), 613-665.

[38] Woronowicz, S. L.: Tannaka-Krein duality for compact matrix pseudogroups. Twisted $S U(N)$ groups, Invent. Math. 93 (1988), 35-76.

revised Dec 10, 1998

Department of Mathematics, University of California, Berkeley, CA 94720

FAX: 510-642-8204

E-mail address: szwang@math.berkeley.edu 